\title{
Gradhiva
}

GRADHIV

Revue d'anthropologie et d'histoire des arts

28 | 2018

Capsules temporelles

\section{Décapsuler. Une histoire vraie}

Decapsulating. A true story

\section{Sara Loar}

\section{(2) OpenEdition}

\section{Journals}

Édition électronique

URL : http://journals.openedition.org/gradhiva/3815

DOI : 10.4000/gradhiva.3815

ISSN : 1760-849X

\section{Éditeur}

Musée du quai Branly Jacques Chirac

\section{Édition imprimée}

Date de publication : 28 novembre 2018

Pagination : 170-193

ISBN : 978-2-35744-111-8

ISSN : 0764-8928

\section{Référence électronique}

Sara Loar, « Décapsuler. Une histoire vraie », Gradhiva [En ligne], 28 | 2018, mis en ligne le 28 novembre 2020, consulté le 07 janvier 2021. URL : http://journals.openedition.org/gradhiva/3815 ; DOI : https:// doi.org/10.4000/gradhiva.3815 

Décapsuler

Une histoire vraie

par Sara Loar

En décembre 1972, avec le lancement de la sonde Apollo 17, la Nasa mettait un terme à son programme d'exploration de la Lune. Lorsque cette même année et la suivante, elle envoya Voyager 1 puis Voyager 2 à destination de Jupiter, Uranus, Saturne et Neptune, l'astrophysicien Carl Sagan demanda de fixer sur les sondes une plaque aux dimensions réduites et très légère, qui a atteint la postérité sous le nom de Golden Record. Pour ce «message à destination de possibles civilisations extra-terrestres ", Sagan et son équipe choisirent cent dix-huit photographies « de notre planète, de nous-mêmes et de notre civilisation ", qu'accompagnaient encore « 90 minutes de la meilleure musique au monde, un essai audio sur l'évolution intitulé The Sounds of Earth, ainsi que des salutations dans une soixantaine de langages humains (et en langage baleine) ».

Imaginons.

Imaginons que l'occasion soit donnée à une anthropologue de rejouer ce geste.

Que choisirions-nous d'envoyer dans l'espace?

Quel serait notre message? Comment nous accorderions-nous sur son contenu?

Qu'est-ce qui, au regard de l'horizon qui est le nôtre aujourd'hui, mériterait d'être sélectionné, transmis? mots clés

archives, collections, bactéries

extraterrestres, patrimoine, muséographie,

Lune, humanité, anthropologie, photographie 
Le vendredi 3 mars 2017 à 14:13:13 UTC+1,

Loar Sara <saraloar@laposte.net> a écrit :

cci: saraloar@laposte.net

Objet: HELP! Fwd: Archives de l'Humanité/Lune

Regardez ce qu'on vient de recevoir!!!

Qu'est-ce qu'on fait?

(Voir ci-dessous.)

Message transféré

Date: 3 mars 2017 à 10:31:31 UTC+1,

De: nicolasdhoc@gmail.com

A: saraloar@laposte.net

Objet: Archives de l'Humanité/Lune

\section{Chère Sara Loar,}

Je me permets de vous contacter suite à la recommandation de M. K. Nous sommes en train de constituer une capsule temporelle comprenant l'ensemble des savoirs actuels de l'Humanité pour les déposer sur la Lune. Ce projet vous évoquera sans doute l'aventure du Golden Record, ce lot de photographies et de sons fixé sur une sonde envoyée dans l'espace en 1972. À la différence du Golden Record, cependant, nous destinons notre capsule non à des extraterrestres mais à nos propres descendants, à un horizon temporel de dix mille ans. La fusée décollera en juillet 2019 de Cap Canaveral. Nous souhaitons ajouter une anthropologue à l'équipe déjà constituée de mathématiciens, biologistes et physiciens, pour rendre compte de la diversité de l'Humanité. Comptant sur votre enthousiasme, je suis disponible pour en discuter quand vous voulez.

Mon numéro: 06 XX XX XX XX.

Bien à vous,

Nicolas

\section{Vertige.}

L'affaire est très sérieuse: Nicolas et son équipe ont prévu d'envoyer, avec la sonde, quatre disques de saphir, quelque mille petits grammes de savoirs constitués qui seront déposés sur la Lune par un robot - un rover dans la vallée de Taurus Littrow. Au sein de l'équipe, j'aurai à charge rien moins que la diversité des cultures... quelques petits grammes rien que pour moi pour saisir l'humanité en quatre mille photos noir et blanc, putativement lisibles à la loupe dans dix mille ans. La raconter, je suppose - donner à tout le moins une idée de ce qui la constituait, de ce qui comptait pour elle.

\section{Vertige...}

La Lune.

Mais la Lune, c'est kitschissime, bon enfant, pudique sur le trivial, sur les affaires de gros sous, les rivalités géostratégiques: une imagerie readymade! C'est Méliès et Cyrano - le vrai et le fictif. Jules Verne. Des chansons 
en pagaille. «Jean de la Lune». L'ami Pierrot. Les sucettes acidulées. Cette boîte de The Scottish Fine Soaps Company, bleu ciel, portant gravée en doré l'inscription «Love You to the Moon and Back», qui m'a fait un clin d'œil sur un rayon du supermarché au retour de mes vacances à Édimbourg, et qui me fait maintenant un clin d'œil obstiné depuis un rayon de ma bibliothèque. Et cætera: tout un menu attirail lunaire m'assaille. Moonboots, moonwalk, des sentences, des histoires grandiloquentes de grands et de petits pas, l'humanité grandie d'un $\mathrm{H}$, la brave Laïka, le cosmoschtroumpf.... Des comics à la pelle, des décorations de nouvel an. La Lune, c'est aussi une playlist: «Moon River», «Back from the Moon ( Michel Benita), David Bowie, Sting...; rien que sur Deezer, pour le mot-clé «moon » il y a plus de trois cents albums, plus de trois cents chansons, plus de soixante-dix podcasts («plus de", ça veut dire que la machine arrête de compter) - et encore, je n'ai pas essayé les autres langues. La Lune, ce truc bête des amoureux... C'est aussi Kim and Buran, les premières raves soviétiques dans des planétariums, au pavillon du Cosmos. L'imagerie soviétique, ces cartes de vœux pour le Jour de la cosmonavigation, l'érotisme étonné des pionnières dans les tableaux tendrement nostalgiques et moqueurs de Dasha Fursey (il faut que je pense à relire ce texte de Yurchak sur la sincérité post-post-communiste, au fait), ces aires de jeux pour enfants représentant des fusées à qui mieux mieux dans les cours des grands ensembles. La conquête triomphante revisitée par les enfants de I'URSS effondrée. Le visionnaire Konstantin Tsiolkovsky (18571935), connu pour avoir dit: «La Terre est le berceau de l'humanité, mais on ne passe pas sa vie entière dans un berceau. »

\section{Mille grammes}

Je commande l'ouvrage - «incontournable", vient de me dire Nicolas au téléphone - de Carl Sagan: Murmurs of Earth. The Voyager Interstellar Record qui retrace la confection express du Golden Record. Voyons voir: comment s'y sont-ils pris? Je tourne les pages qui, suivant le mot de Danowski et Viveiros de Castro, fleurent bon «l'épopée de l'Esprit », la fidèle compagne de tout projet de conquête un tant soit peu affermi auquel on aurait enlevé son point d'arrêt - c'est cela, précisément, qui me rend a priori le geste sympathique: la sonde et le disque continuant de dériver dans l'espace à l'heure où j'écris ces lignes (aux dernières nouvelles, Voyager 1 est à plus de vingt milliards de kilomètres de la Terre, talonné à trois milliards de kilomètres près par Voyager 2...). Cependant, mes sentiments se mitigent à mesure que je parcours l'ouvrage. Pourquoi des énoncés du type "des vaisseaux comme émissaires de la Terre dans le royaume des étoiles" me laissent-ils un goût suspect? Le long article produit par Sagan lui-même est intitulé «For Future Times and Beings». Sans surprise pour l'époque, le débordement de soi et de son présent, de son immanence, est donné comme salutaire - parce qu'il est exploratoire et que l'exploration compte au motif des accomplissements louables. Je pense: parfois, ce débordement peut se dire aussi comme une nécessité. Je pense: enfouissement des déchets nucléaires pour lesquels on doit encore trouver, pareillement, le moyen de faire perdurer un message... Je pense à John D'Agata, à Yucca Mountain dans lequel il spécule - non, décrit - ce qui peut arriver à cette idée savante d'une pancarte qui signalerait, à l'aplomb du site d'enfouissement, le legs dangereux, mortifère. La pancarte dirait quelque chose comme: 
"Nous ne commémorons aucun acte d'importance. Aucun objet de valeur n'y a été enterré. Ce lieu est un message et fait partie d'un système d'information. Faites attention. Nous sommes sérieux. Nous avons jugé important de vous communiquer ce message. Nous pensons que notre culture a eu de l'importance » (p. 88), mais dix mille ans après:

"La langue utilisée dans ce message n'aurait conservé que $11 \%$ de sa signification, et donc de son sens, et donc de sa capacité [...] à exprimer quoi que ce soit. » (p. 89)

«Nous

de

message système

Nous

Pour les déchets à enfouir comme pour les images à envoyer sur la Lune: penser le message à dix mille ans... Qui le lira et que liront-ils? Quelle est au juste la «durée de vie d'un fait» (l'autre livre que John D'Agata a écrit avec son fact-checker, Jim Fingal)? Et en quelle langue, en quel langage commun, l'inscrire? Comment un message extrait de son lieu, de son temps d'énonciation, peut-il continuer à porter un sens, à valoir comme acte proactif de communication?

Sagan et ses collègues se sont bien sûr penchés sur la question. Le problème de la criticité du message en moins, ils ont convenu d'un certain nombre de préalables à leur entreprise. Dès la page 3 du livre, ils imaginent que le futur sera différent de ce que nous connaissons, mais que ceux qui le peupleront voudront savoir qui nous avons été - de la même manière que nous cherchons à connaître ceux qui nous ont précédés. Quels qu'ils soient, on peut compter sur leur curiosité, à tout le moins sur leur appétence historique: ceux-là voudront entendre et même comprendre le message dont les disques sont porteurs. Ils sont déjà virtuellement équipés pour ne pas faire des disques dorés des dessous de plat, des cales pour vaisseaux ou des frisbee, voilà qui est rassurant.

Bien. Le contenu du message, ensuite. En la matière, j'envie presque la foi de Sagan et de ses coéquipiers en la "Science», qui leur fait dire, trois pages plus loin: "S'il est possible de communiquer, nous pensons que nous savons sur quoi porteront les premiers échanges: ils porteront sur la chose que les deux civilisations ont l'assurance de partager, la science. " L'énoncé a lui-même une prémisse très puissante: "Comme nous, Ceux-là doivent se coltiner les mêmes lois de la physique, de la chimie et de l'astronomie.» (p. 5) La nature, cette extraordinaire ordonnatrice et pacificatrice, étend ses pouvoirs bien au-delà de notre système Terre (le Face à Gaïa de Latour) et c'est la «réalité extérieure" - l'irréductible composition des étoiles et leurs propriétés spectrales, par exemple - qui tisse, entre Eux et nous, ce lien inaltérable: non seulement lls se montreront attentifs aux disques et chercheront à en décrypter le message, mais en plus lls en ont potentiellement la clé...

Cette confiance, qui semble d'abord sans limite, rencontre cependant ses points de butée qui, je trouve, rendent l'entreprise attachante. Par exemple, c'est peu dire que Sagan et son équipe affichent d'abord une confiance dans 
le langage mathématique, le seul à répondre au «besoin impérieux de clarté absolue " (p. 66). Et j'imagine bien l'affreuse désillusion - le «choc déprimant» (p. 53) - de Frank Drake lorsque, sur l'ensemble des membres d'un comité d'experts constitué ad hoc auxquels l'astrophysicien soumet son message de 55-caractères-produit-de-19-et-29-qui-sont-des-nombres-premiers, aucun n'est capable de l'interpréter... Le décollage n'a pas encore eu lieu, la rencontre est encore loin devant que déjà le langage mathématique se retrouve échec et mat. De quoi donner du fil à retordre à Meillassoux: I'axiome suivant lequel «dans le futur anticipé il y aura encore possibilité de soumettre le monde à une connaissance scientifique " est déjà en train de vaciller...

Récapitulons. Eux et Nous, soit une même physicalité. Une envie de savoir. Une démarche de connaissance. Une volonté d'explorer. Eux et Nous formant un «Nous » unique et parfaitement œcuménisé comme aurait pu dire Istvan Praet... Ooohh non! Se pourrait-il que les Lointains de l'espace et du temps soient des conquérants comme nous?

\section{Ceux qui restent}

Golden Record raconte une épopée avec l'Humanité comme héroïne; la conquête spatiale est sa scène et la Science son bras armé. Du moteur à songeries au trophée d'humanité... Comment vais-je m'en sortir, moi? Et je vais les trouver où, les quatre mille photos? La question me brûle les lèvres, j'ai envie de la poser à tout le monde - autour de moi, dans le métro, au supermarché: et vous, qu'enverriez-vous sur la Lune?

Dans le livre édité par Sagan, Jon Lomberg, l'artiste qui a travaillé à la sélection des photographies pour Golden Record, s'interroge aussi: (qu'estce) qui embarque? La question s'affine en considérant d'abord son négatif. (Qu'est-ce) qui reste? Opération de qualification/disqualification: il y a ce qui gagne une place au soleil de la postérité et le reste. Le reste, c'est par exemple la guerre, la maladie. Par exemple le crime et la pauvreté: « Nous avons décidé que le pire de nous-mêmes n'avait pas besoin d'être envoyé à travers la galaxie.» (p. 76) Ou encore la religion. Trop compliquée. En représenter une obligerait - par une pirouette diplomatique qui ne vaut étrangement ni pour les hommes, ni pour les animaux, ni pour les systèmes politiques, etc. à représenter toutes les autres et on en oublierait nécessairement. De même, l'art (quand bien même la piste sonore contient des morceaux de Mozart). Trop subjectif.

L'espace ne saurait donc servir de réceptacle qu'au meilleur de nousmêmes, qu'à ce qui nous définit sans équivoque. Je n'avais pas ça en tête. Les gens à qui je pose la question non plus, manifestement. Autour de moi, les réponses s'égrènent: des photos de ses propres enfants, des témoignages du désastre écologique, le dodo de l'île Maurice accompagnant tous les existants disparus depuis, le continent de plastique... Survivre, disparaître: voilà ce que suscite aujourd'hui une projection à dix mille ans.

Je prends note, démarre une collection. J'ajoute des photos de Shoah, de Rwanda, de fosses communes, de tout ça. Mais je bute sur la montée en généralité, il y a un degré d'abstraction qui ne peut pas marcher: à chaque absolu de l'horreur et du malheur humains, il faudrait pouvoir rendre son absolue singularité. Et puis il y a ce sentiment de malaise - une capsule temporelle 
pour nous dans dix mille ans: à quoi bon? Le futur, nos descendants dans dix mille ans, dans le fond, je m'en fiche. Le rêve de Lune, il est déjà derrière nous. À propos du rideau de fer et de l'impossibilité de le franchir, Svetlana Boym a déjà écrit The Future of Nostalgia: «Il semblait que nous irions sur la Lune plus vite qu'à l'étranger. » (p. 60) L'URSS s'est déjà effondrée. J'ai déjà appris à taper à la machine sur le très besogneux clavier QWERTY d'une machine mécanique hors d'âge, dans cette église de Kitaï-Gorod reconvertie en cours Pigier soviétique, dactylographes, tractoristes et travailleurs de tous les pays - uniformément mais sûrement - unis dans la gloire au travail socialiste. Souvenir, souvenir: cet exercice du 16.03.92, Groupe 37 :

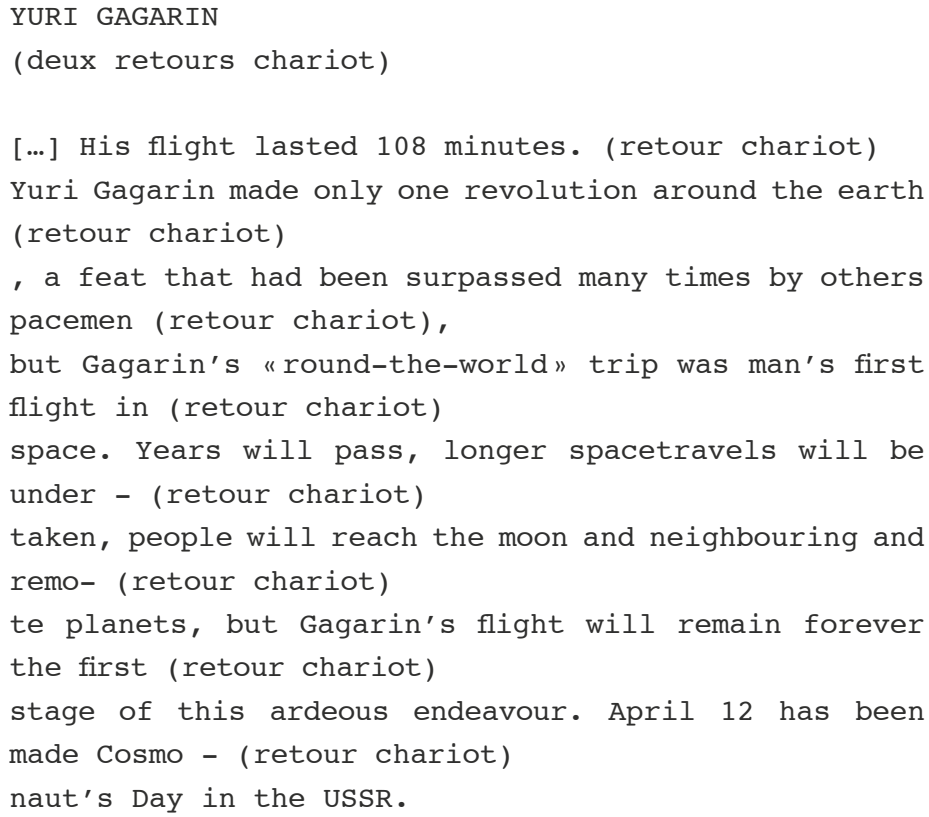

Deux fautes de frappe. La Lune, c'est has been, retour chariot.

Et puis je n'y crois pas trop qu'on sera encore là dans dix mille ans pour aller sur la Lune et, après tout, l'extinction de l'humanité me laisse plus dubitative qu'éplorée. En fait, on va tous mourir et ce n'est pas très grave; et en fait je suis certes anthropologue, mais tout d'un coup je ne suis plus très sûre d'être philanthrope. II suinte l'angoisse, le projet, je trouve. II a un côté morbide. Plus morbide qu'optimiste, ou trop forcené dans l'optimisme: un peu malsain. J'écoute d'une oreille distraite une émission de radio sur le dernier Français condamné à mort et décapité. Soudain, tout s'éclaire. Dernière cigarette, dernier cognac et dernières paroles avant décollement. C'est exactement l'exercice qu'on me demande.

Voilà, c'est malin: cette série d'interrogations tourmente mes journées, je ne regarde plus la Lune du même œil. C'est cette même interrogation inquiète, très fin de monde, qui teinte Fahrenheit 451, le film de Truffaut, dans l'ultime scène de la communauté des «hommes livres » qui apprennent par cœur des livres pour en garder la mémoire. C'est cette interrogation encore que dépeint Jean Hegland dans son roman, au moment poignant où les deux sœurs s'apprêtent à quitter leur maison et à y mettre le feu, pour 
gagner définitivement la forêt. Fin de monde prématurée, bifurcation sans appel, à laquelle pendant longtemps elles n'ont pas cru et qui les a rattrapées. C'est l'heure de faire leurs adieux à l'état des choses tel qu'elles le connaissaient: «J'ai essayé désespérément de réduire le nombre [des] livres absolument nécessaires. Mais étalé par terre, chaque volume était sa meilleure défense. Tous semblaient incomparablement précieux. Comment pouvais-je décider que les Poésies complètes d'Emily Dickinson valaient plus que les Contes de Grimm, ou qu'il fallait renoncer à L'Origine des espèces au profit du Troisième Reich, des origines à la chute: une histoire de l'Allemagne nazie? Un instant il m'a semblé plus équitable, peut-être même plus charitable de les brûler tous. » (p. 298).

Retour chariot.

L'horizon, de plus en plus tangible, de notre finitude vient brouiller les lignes du message, qui ressemble de plus en plus à une bouteille à la mer, un geste ultime de survie - par délégation, en quelque sorte. Ça change la donne... Que décide-t-on de sanctuariser? À quoi réellement tenons-nous? De quels objets faire nos dignes représentants, lesquels auront pour mandat notre pérennisation?

\section{Encapsuler l'humanité}

Soirée de discussion avec les collègues. J'en sors confortée dans mes doutes, mais ils confirment aussi que le devoir m'appelle, qu'il serait irresponsable de refuser une proposition pareille. Mais pourquoi une anthropologue, pourquoi moi? La question revient, lancinante, ça gratte un peu. Nicolas, d'emblée, a été très clair: anthropologue ce sera, because "diversité culturelle". C'est son domaine de compétence à l'anthropologue la diversité culturelle, son créneau, son petit pré carré - pas grand, mais bien carré, le pré. Les anthropologues n'ont-ils pas vocation à «tailler dans la diversité des expériences du monde des pièces de même format [...] afin de les disposer sur le grand lé de la nature", à juxtaposer le «bariolage de couleurs, de motifs et de textures " (dixit Descola dans sa Leçon inaugurale), des manières de se coiffer, de danser, d'habiter? Retour chariot.

Je songe au temps où, apprentie ethnologue sur les bancs de la fac, I'histoire de l'anthropologie m'était contée à grands pas, à grands sauts de paradigmes. Je me souviens du projet intellectuel de rendre compte de l'homme dans toutes ses dimensions et sous toutes les latitudes, d'embrasser tout le cycle des savoirs disponibles, d'en faire le tour, qui a bien occupé la seconde moitié du XIXe siècle. Mais l'utopie de constituer les "archives totales de l'humanité", selon les termes de Griaule, s'accompagne d'une focalisation sur les objets, leur collecte systématique, le désir d'inventaire encyclopédique du monde (il faudra que je relise Benoît de L'Estoile). Sur le modèle des inventaires d'espèces, les outils, les masques, les mythes, les photos, les diagrammes de parenté, rassemblés dans les lieux de stockage et d'accumulation que sont les musées d'ethnographie d'entre-deux-guerres, font figure de spécimens à décrire, pour donner à voir les cultures comme des ensembles constitués et répertoriés. Un monde fini, dénombrable, typologisable en somme avec ses unités idiosyncratiques. Puis le tournant malinowskien, fondant un nouveau rapport à la culture, 

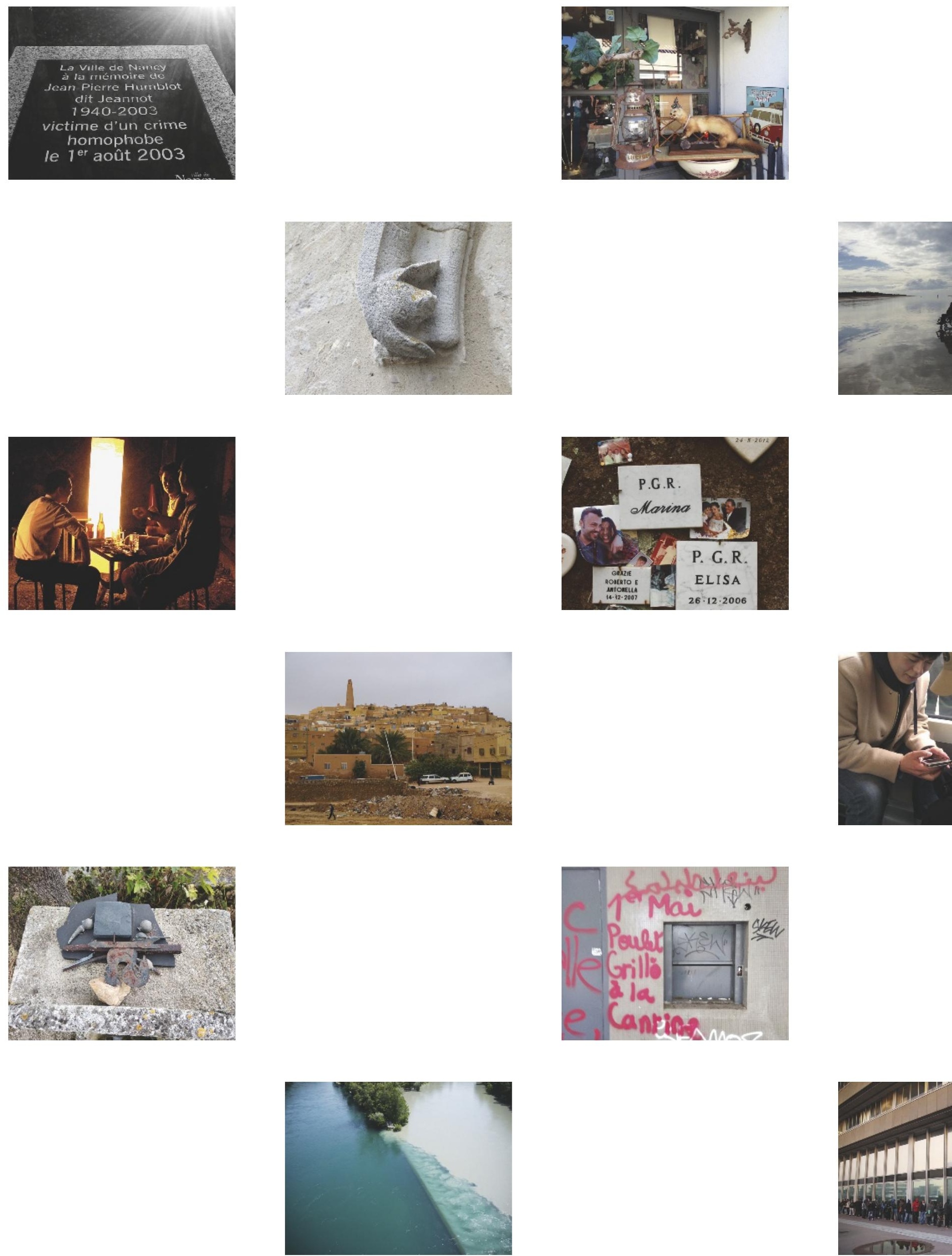

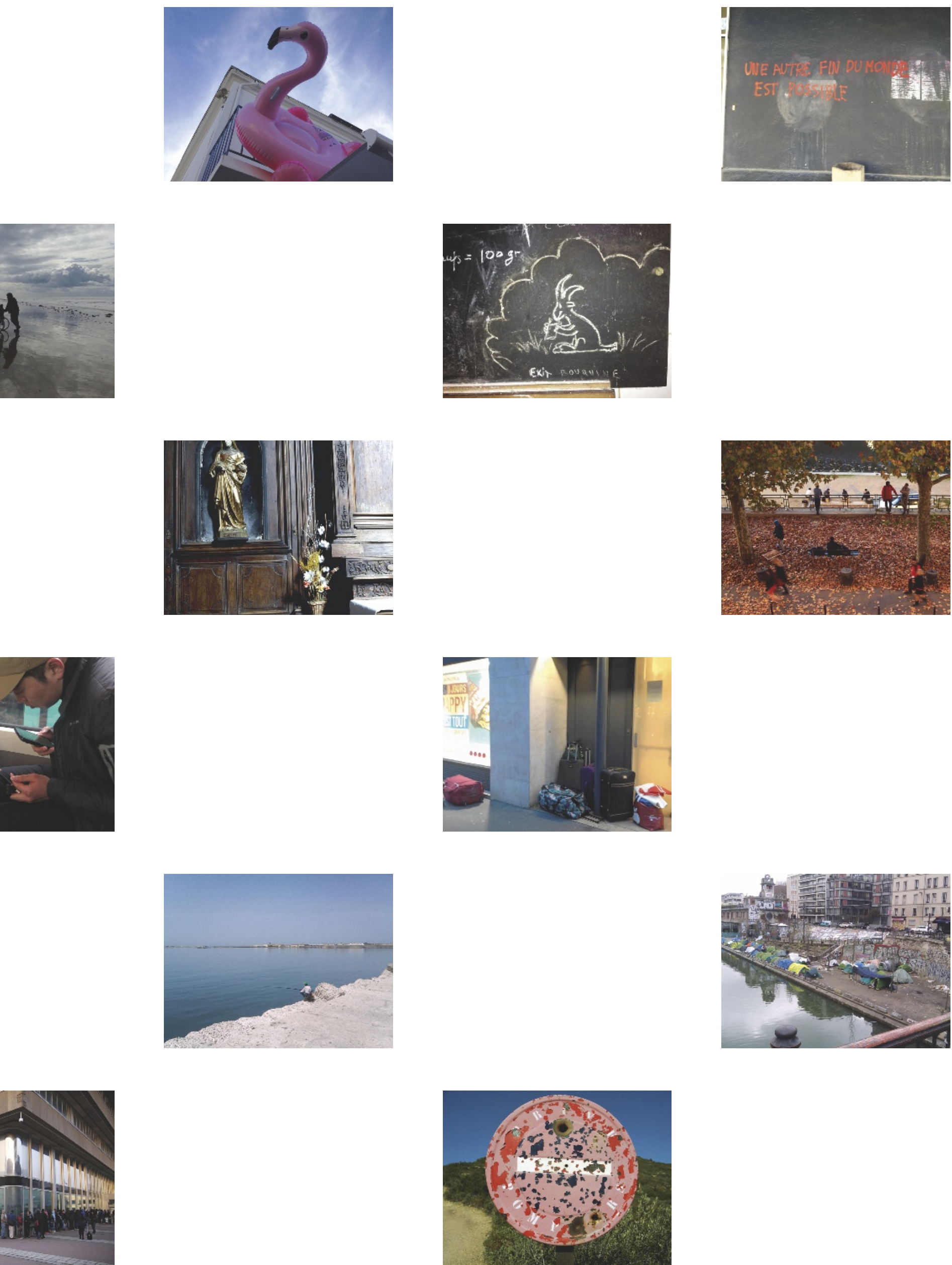


\section{double page précédente et p. 186-187}

La personne qui reçoit les photos travaille à mettre en écho ces images avec d'autres de son cru et les envoie à son tour au suivant. plus intime, plus engagé, plus interactionnel, pour décrire cette fois un «homme total» sur les pas de Marcel Mauss, le «canoë total »... Savoir encyclopédique, puis savoir symbolique: comme si la connaissance anthropologique ne pouvait faire autre chose que de reposer sur des mondes à comparer toujours et faire exister comme totalité signifiante. Des cultures, des amalgames, des précipités, des concrétions...

Le Golden Record posait comme l'un des principes, destinés à rendre opératoire et surtout significative la sélection, d'« envoyer l'information concernant la Terre et ses habitants que les auditeurs extraterrestres ne trouveront pas autrement". "Plus l'information est spécifique à la Terre, écrivait Sagan, plus elle est anecdotique et idiosyncratique, et plus il sera difficile pour les extraterrestres de la comprendre - mais en même temps plus cette information, une fois comprise, aura de valeur. » (p. 33)

II y avait bien là une piste, susceptible d'éloigner avantageusement de l'autoroute de la représentativité et du comparatisme étroit, en choisissant de frayer plutôt avec des intimités irréductibles. Mais l'équipe avait probablement sous-estimé les effets induits par la pauvreté des moyens ou par les projets toujours plus courts, leur accélération inconséquente ou leur complication administrative. Pour des questions de copyright, c'est à la National Geographic Society qu'elle a recours... La NGS «qui, à sa façon, faisait systématiquement et à grande échelle ce qu'on essayait de faire: donner une image totale de la Terre et de ses habitants », notait Sagan (p. 77).

La National Geographic Society, donc. Et si je reprenais le problème en partant du format photographique? Rendre présent un objet absent et imiter son apparence, rendre sensible: les images comme mode de représentation du réel semblent toujours s'imposer, quand vieux de dix mille ans et nous adressant à des inconnus, habitants ou de passage sur la Lune, nous devons témoigner du monde terrien que nous habitons et parcourons. Pourquoi ce choix de l'image photographique comme support documentaire privilégié ? Comme si nous étions condamnés à inlassablement rejouer Voyager, jusque dans son format...Des photos, des photos... Mais, moi, j'ai en tête Lorraine Daston et Peter Galison, et comment la photographie ne s'est imposée comme forme par excellence de documentation du monde - sa reproduction exacte - qu'en dénouement d'un long processus, à la fois moral et technique, d'objectivation mécanique. Et assez paradoxalement, la fixité de l'image rend pourtant impossible la saisie même de ce qu'est la vie. II faudrait que je retrouve ce passage où Tim Ingold remarque que la vie est mouvement, exploration, transformation, animation - soit l'exact opposé de la fixation qu'opère l'image. L'engouement pour la photographie comme procédé technique d'archivage du monde a certes d'abord procédé de considérations matérielles - mettre le monde à plat, le découper en rectangles, le ranger bien propre dans des boîtes, c'est pratique. On pourrait pourtant imaginer d'autres formats, d'autres manières d'encapsuler notre monde... S'il ne tenait qu'à moi, vraiment, à l'heure qu'il est, j'enverrais sur la Lune une bulle d'air, qu'il faudrait encore choisir, sélectionner, singulariser (un wagon du RER A aux heures de pointe? un vieil appartement enfumé du Caire?), un peu comme ces scientifiques dont William Jarvis rapporte comment ils s'évertuèrent à produire, à l'intention des savants futurs, des capsules d'atmosphère: en somme, une archive discrète de l'humanité. 
Que laisser sur la Lune, des images traces et vestiges, des images artefacts, des images témoins, des images signes ou messages? Un peu de tout ça sans doute, mais ça fait quand même beaucoup! Et pour former quel ensemble? Comment faire tenir les images et ce qu'elles représentent? Par jeux d'affinités, de ressemblances, d'analogies, de contrastes? Retors, mon esprit dérive, s'amuse à imaginer une juxtaposition d'hapax, véritable pied de nez à la connaissance. Dans tous les cas, échapper à tout prix au classement thématique des réalités du monde, au miroir déformant tendu à nos futurs «Spectator » - il faut que je relise Roland Barthes! - d'un monde catégoriel tiré au cordeau, évidé de ses incertitudes, béances de sens, chausse-trappes. Je ne renonce pas à explorer ou à imaginer autant d'histoires que d'agencements et de mondes relationnels dont l'ordonnancement, incertain, reste à qualifier, et qui résiste.

Si mon seul médium pour entrer en communication avec les potentiels destinataires, ce sont des images, alors c'est bien leur agencement que je peux travailler. Je me demande quelle aurait été la texture du monde embarqué et encapsulé dans la navette Voyager si d'autres économies de la connaissance, d'autres modalités de produire, de choisir, de trier, d'archiver, d'agencer les images avaient été retenues.

\section{Accumuler}

Le 19 juin 2017 12:44:31 UTC+1, <laure.hélaille@gmail.com> a écrit:

\section{Bonjour Sara,}

Tu vas bien? Je t'écris pour savoir si tu aurais un peu de temps mardi prochain avant le séminaire pour discuter avec $\mathrm{H}$ (en copie) et moi. $\mathrm{H}$ est actuellement en stage pour la revue. Elle s'occupe plus particulièrement, dans ce cadre, d'un partenariat avec le musée Albert Kahn. On est en train de défricher un peu tout cela, et on voulait te solliciter sur deux aspects:

- la question de l'archive: le musée a un fonds d'archives assez impressionnant, notamment photographiques et filmiques, dont une partie est numérisée et le reste est en train de l'être. On cherche donc quelqu'un qui accepterait d'écrire un billet de blog sur la question du statut de l'archive... et on a pensé à toi:). Mais si tu n'as pas le temps, ou que tu as d'autres idées, on est aussi preneuses de conseils.

- idem pour la question du portrait, qui constitue une grande partie des fonds photographiques du musée.

$\mathrm{H}$ pourra t'expliquer tout cela mieux que moi, car elle a rencontré la directrice il y a une semaine.

Qu'en penses-tu? Serais-tu disponible pour prendre un café avec nous mardi prochain vers $15 \mathrm{~h}$ ?

Bises,

Laure

Je consulte distraitement le site Web du musée Albert Kahn, et tombe sur les Archives de la planète (ADLP), soit un fonds de dizaines de milliers de photographies, prises entre 1913 et 1946 par des «opérateurs» qui ont été envoyés pour, littéralement, arpenter tous les recoins du fait humain. 
Là encore, il est question d'enregistrer ce qui va disparaître, et en particulier ce monde rural, méconnu des élites, appelé à s'effacer devant l'industrialisation triomphante: à l'origine des ADLP, je trouve Albert Kahn, banquier, mécène, philanthrope, rêvant d'un humanisme absolu, reposant sur la collaboration pacifique entre les peuples et une connaissance fine de la diversité humaine. De ce que j'en lis dans le catalogue d'exposition consacré au projet par le musée, Kahn s'empare de l'avènement de la technique photographique pour sauvegarder les «pratiques et modes de l'activité humaine », en fixer les traits de manière pérenne, donner à voir un "état du monde» à l'intention des générations futures. D'après Marie-Claire Robic, le projet de Brunhes est celui au fond d'une "géo-photo-graphie» dans lequel l'outil photographique - des autochromes, une technique particulièrement lourde et coûteuse - est mis au service de la capture, dans ses plus petits détails, d'une humanité ordinaire prise sur le vif. Les opérateurs, que Brunhes lui-même forme à la prise de vue photographique, sont dotés d'instructions précises sur les cadrages, les sujets: toujours prendre les humains dans leur environnement. Privilégier le «typique», dit Brunhes, c'est-à-dire ici le banal, l'ordinaire, «l'agglomération anonyme» qui ne se remarque pas. Surtout ne pas trier, ne pas sélectionner, encore moins hiérarchiser; mais entasser, agglomérer, juxtaposer ces prises de «petites foules » dans une joyeuse frénésie, tel semble être le credo des Archives de la planète.

Plus tard dans l'après-midi. J'évoque avec Vincent D. le projet Kahn et tandis que je lui parle du chapitre de Teresa Castro à ce propos dans Les Lieux de savoir, il me signale un autre de ses articles, qui porte sur les films également tournés par les opérateurs des ADLP. D'après Castro, les films étendraient l'ambition descriptive théorisée par Brunhes de saisir le monde humain au plus près. Expérimentation d'un cinéma documentaire alors dans ses prémisses, les films tournés pour les ADLP résistaient à la coupe, au montage, au spectaculaire, privilégiaient les plans-séquence bruts, lents et longs, tournés à hauteur d'homme.

Ce que dessinent les images produites pour les Archives de la planète est, au fond, le portrait luxuriant et pléthorique d'une humanité à ras de terre. Qu'aurait donné le Golden Record s'il avait pris comme modèle les Archives de la planète plutôt que le National Geographic? J'imagine, à rebours de photographies stylisées ou de tableaux d'une humanité hégémonique - dont cette photographie d'un groupe d'enfants tenant, littéralement, le globe terrestre dans la main est peut-être l'expression la plus achevée des hommes fondus dans leur environnement quotidien; des clichés dé-centrés, des humanités aussi foisonnantes que furtives.

\section{Puzzler}

Ou encore: par une autre opération d'amoncellement de choses et d'images, faire jouer les variations, non pas les grands écarts pour donner à voir le monde bigarré, mais les micro-événements, l'infime, qui se décline et dont le rapprochement crée des continuités faisant apparaître le changement. Je vois, à la manière de Georges Perec arpentant la rue Vilin d'année en année, des captations vidéo de paysages urbains ou ruraux, ou périurbains, pris au même endroit, avec le même point de vue, de saison en saison, d'année en année, de décennie en décennie. Sur ce que l'on perd... mais aussi de quoi l'espace se peuple au fil du temps. 
À l'inverse du propos encyclopédique qui nourrit les Archives de la planète, le principe de la série s'extrait du principe exhaustif pour dire le lien. Si je pense par série, je pourrais par exemple jouer jusqu'à l'absurde du principe de juxtaposition qui a donné naissance à la photographie d'identité - ça me rappelle Alfred Batut qui superposa inlassablement des photographies de visages capturés à distance et angle égaux pour tenter d'obtenir des «types ethniques génériques "...

Le travail par affinité, par «airs de famille », qui caractérise la série peut cependant être subverti par le principe du puzzle, dont Perec toujours, qui fit de l'archivage son métier, nous dit, en préambule de La Vie mode d'emploi: "On peut regarder une pièce [du puzzle] pendant trois jours et croire tout savoir de sa configuration et de sa couleur sans avoir le moins du monde avancé: seule compte la possibilité de relier cette pièce à d'autres pièces, et en ce sens il y a quelque chose de commun entre l'art du puzzle et l'art du go; seules les pièces rassemblées prendront un caractère lisible, prendront un sens: considérée isolément une pièce d'un puzzle ne veut rien dire; elle est seulement question impossible, défi opaque; mais à peine a-t-on réussi, au terme de plusieurs minutes d'essais et d'erreurs, ou en une demi-seconde prodigieusement inspirée, à la connecter à l'une de ses voisines, que la pièce disparaît, cesse d'exister en tant que pièce: l'intense difficulté qui a précédé ce rapprochement, et que le mot puzzle - énigme - désigne si bien en anglais, non seulement n'a plus de raison d'être, mais semble n'en avoir jamais eu, tant elle est devenue évidence: les deux pièces miraculeusement réunies n'en font plus qu'une, à son tour source d'erreur, d'hésitation, de désarroi et d'attente. » II poursuit un peu plus loin: "Ce n'est pas le sujet du tableau ni la technique du peintre qui fait la difficulté du puzzle, mais la subtilité de la découpe..." Puzzler embrasse un double geste: reconstitution de fragments dispersés de manière aléatoire dans le site archéologique de l'humanité et travail de découpe, geste d'orfèvre pour fragmenter notre archive selon des lignes sinueuses que mon destinataire devra recomposer à l'issue d'un patient jeu de rapprochements.

\section{Abréger}

Rêve, cette nuit, d'un atelier clandestin de fabrication d'objets de contrefaçon installé dans un appartement haussmannien, débordant de pièces de tissu, d'objets manufacturés, de rebuts prêts au recyclage, d'outils de travail du cuir, du cuivre, du bois, d'affiches aux murs, de femmes et d'hommes, nés en différents lieux de la planète, penchés sur leur établi, et un point de vue sur la rue depuis les fenêtres. À ajouter à la liste: l'amoncellement. Ici, l'amoncellement fait monde par la coprésence d'êtres et de choses en circulation et en transformation, et par celle d'images à imbriquer. Je feuillette Le Livre des merveilles (ou Le Devisement du monde) de Marco Polo, une vraie plongée dans la sidération des savants de la fin du XIII siècle en Europe confrontés à l'ailleurs. Dans les cabinets de curiosités ont proliféré, dans un savant désordre, mappemonde, squelettes, antiquités, médailles, dents de géant, cornes de licorne, poudre de momie, aberrations de la nature, animaux empaillés, artefacts insolites. L'art et la nature - artifialia et naturalia étaient convoqués ensemble, sur le même plan, pièce à pièce, avec leurs défaillances ou leur perfection. On est bien loin ici du projet encyclopédique! Pôle d'érudition, les rassemblements hétéroclites dévoilent les secrets intimes de la technè, sans chercher à s'ancrer dans les grands projets 
de classification universelle qui mobilisaient les humanistes à la même époque (je dois en reparler avec Patricia Falguières). Dans les chambres closes, les choses remarquables composent un microcosme, non pas un monde en condensé, mais un amoncellement du divers, un «abrégé », disait Antoine Schnapper, où l'exemplarité l'emporte sur la représentativité, des choses que la pensée, trop occupée à en débusquer les excentriques spécificités, ne s'attarde pas à relier ou classer. À la manière de certains artistes contemporains comme Mark Dion, adepte du brouillage des oppositions entre nature et culture, vrai et faux, science et art, je pourrais envoyer sur la Lune la photo d'une vitrine exposant pêle-mêle débris organiques et déchets manufacturés issus de la fouille d'une mare asséchée. Histoire partiale et partielle, certes, mais puissant abrégé de ce que Frédéric Joulian et Agnès Jeanjean appellent «la société par ses restes».

\section{Démonter}

Près de cinquante ans après, c'est fou comme le projet de Sagan paraît marqué par une volonté de clarté, de transparence du message et des images, mais aussi de linéarité. Je viens de comprendre que les images présentes sur le Golden Record ne s'y trouvent que sous une forme codée, linéaire, qui fait de ce disque un objet étonnamment proche des vinyles de l'époque. À supposer que les lecteurs du futur et de l'espace soient assez malins pour déchiffrer le disque de Voyager, pour transformer la piste en images, à supposer tout ça, ils n'auraient devant les yeux qu'une suite de clichés se donnant dans un ordre autoritaire et sans joie.

Sagan avait raison: quitte à psychologiser les extraterrestres, autant penser qu'ils seront non seulement concernés par la Science, mais aussi excités par les mêmes choses que nous. Or rien n'ennuie plus les chercheurs que les discours transparents, limpides: les meilleurs articles, les meilleures enquêtes et les meilleures controverses sont produits pour résoudre un piège, une énigme tendue par d'autres. On ne compte plus les interprétations

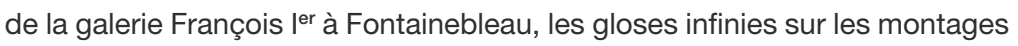
savants de Aby Warburg... C'est peut-être comme ça qu'il faut procéder, non pas expliciter, mais ralentir, piéger, créer non seulement de l'énigme, mais du désir d'enquête.

Justement, des planches de Mnémosyne de Warburg aux atlas de Georges Didi-Huberman, les propositions de montage cherchant à rendre compte de l'hétérogène, à “échantillonner le chaos ", n'ont pas manqué de théorisations ou de propositions depuis les années 1970. C'est seulement si on arrive à créer un dispositif où les images s'enchaînent autrement que dans une pure juxtaposition qu'on peut avoir une chance d'embarquer nos interlocuteurs du futur dans les détours des expériences de pensées du début du $x l^{e}$ siècle. Devant l'arbitraire absurde de la sélection, de l'élection de quelques centaines ou milliers d'images pour rendre compte de l'ensemble des expériences terrestres du temps et de l'espace, la seule solution est d'indiquer que l'intéressant ne se trouve pas dans les images mais entre les images. C'est bien par un travail de montage (en pariant sur le démontage du futur) qu'on peut rendre compte, donner à sentir quelque chose de la vie sociale, de la vie tout court, sans courir le risque d'essentialiser quoi que ce soit. 
II s'agit en l'occurrence d'un type de montage bien particulier qui aurait comme principale vocation de donner à penser que, dans ce cas, le vide entre deux images est d'une épaisseur inouïe.

II faudrait dès lors prendre la surface du disque lunaire comme un véritable plan d'immanence, une surface dont ni le centre ni la périphérie ne seraient valorisés, sur laquelle on laisserait les images proliférer, se fiant à leur valence quasi végétale... Mais comment agencer l'hétérogène quand tout se trouve écrasé, mis en forme dans le cadre contraignant de l'image en deux dimensions monochromes? Une possibilité pourrait être de laisser s'y déposer un peu de temps sur Terre, de ne pas travailler dans l'urgence qui a poussé Sagan à faire ce qu'il pouvait. On pourrait ainsi imaginer un mur d'images qui ne serait pas un ordonnancement pensé par une seule personne ou presque, fût-elle géniale (comme c'était le cas avec Warburg), pas plus qu'il ne serait la traduction d'un jeu entre anthropologues choisis. On pourrait imaginer que ce montage d'images s'établisse dans le temps, pendant des années, entre anonymes, un peu à la manière de ces murs d'ex-voto qu'on peut voir dans les rues de Rome, où un témoignage du xIxe siècle est recouvert par une déclaration d'amour de 2010, où un désir dans les années 1920 sert de support pour un passant des années 1970 qui vient y inscrire sa propre demande, son propre souvenir. Jeu de montage à travers le temps entre anonymes, notre mur d'images devrait donner du fil à retordre et pas mal de plaisir aux Sélénites à venir.

\section{Bouts de ficelle}

Le 20 novembre 2017 à 13:37:04 UTC+ 1, Loar Sara <saraloar@laposte.net> a écrit :

Chères amies, chers amis,

${ }^{*}$ WARNING Ceci est un long mail, installez vous confortablement!!!**

Pour ceux qui n'ont pas pu être là, $C R$ de la réunion d'hier soir: plutôt, comme il nous est suggéré par l'équipe de Nicolas, que de plonger tête la première dans des banques d'images qui risquent d'être indigestes (National Geographic, CERN, CNRS, etc.), nous est venue l'idée d'entamer, un coup pour voir, une sorte de cadavre exquis (plus poétique qu'une chaîne de l'amitié!) d'images, que nous irions à tour de rôle chercher dans nos images personnelles, des images ou visuels libres de droit, et aussi que nous produirions pour l'occasion. Le jeu a quelques contraintes: quatre axes ont déjà été sélectionnés par l'équipe, qui ont été conçus pour croiser les données de chacune des disciplines, et en ce qui me concerne, j'y vois un moyen de faire la peau à la partition avec laquelle ils travaillaient jusqu'alors, puisqu'ils avaient imaginé d'abord de consacrer le gros des disques en saphir aux données "naturelles ", disons, et d'en laisser un à disposition pour la "diversité culturelle». Donc on croise et on mélange tout, finalement, sur la base de quatre entrées (qui ne vous paraîtront pas totalement étrangères, je pense...) qui vont fournir le socle de nos «histoires 

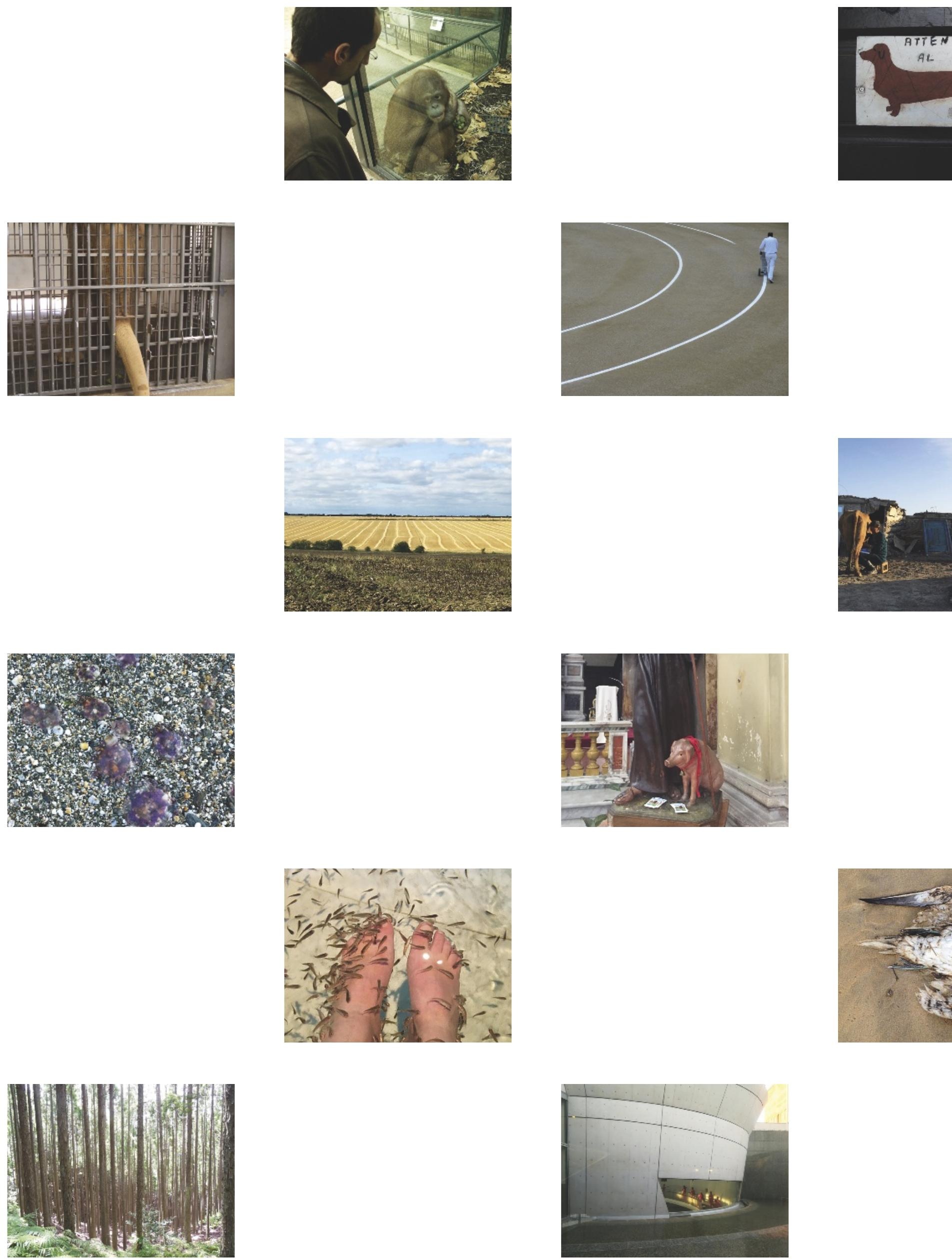

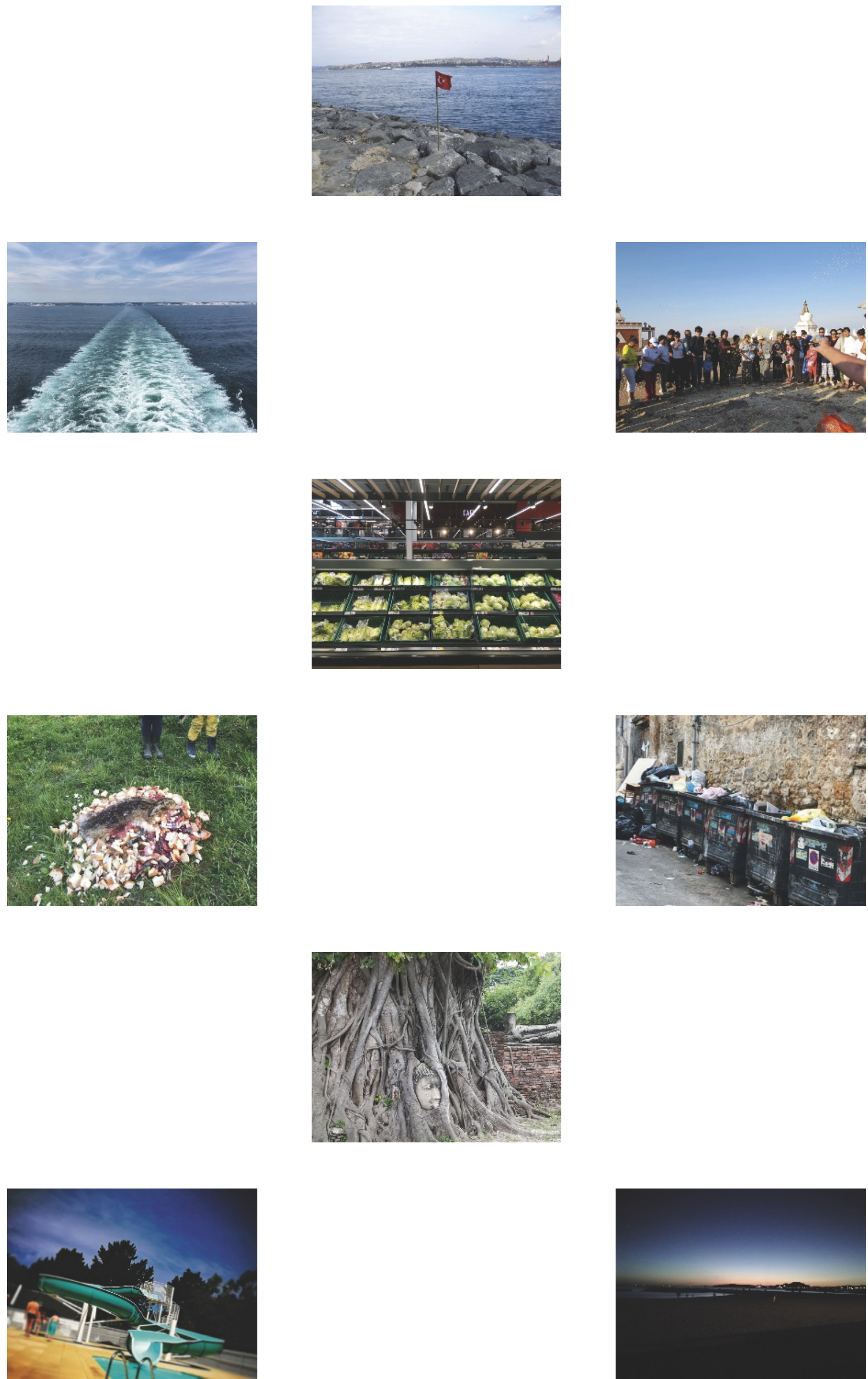
photographiques »: AIR/TERRE/EAU/FEU. Sans que ce soit décisif pour la suite, il nous a semblé que ces histoires pouvaient servir à enfiler des images comme des perles. Nous sommes sept, à chaque entrée correspondra une boucle. Je lance une première image ou une série d'images, que j'envoie à la personne après moi dans la boucle - le geste d'adresse est important. La personne qui reçoit travaille à mettre en écho ces images avec d'autres de son cru et les envoie à son tour au suivant, etc. Pour le moment, on essaye simplement, en usant des moyens technologiques usuels, mais on a été assez loin dans l'affinage du dispositif en imaginant qu'on pourrait trouver une salle quelque part (par exemple au musée du quai Branly), une salle et quatre murs sur lesquels on viendrait punaiser à tour de rôle nos images en envoyant un signal de fumée (je plaisante, un texto ferait l'affaire...) aux autres pour les informer que quelque chose de nouveau les attend là. Mais en attendant de pouvoir mettre en œuvre cette idée, qui permettrait d'exposer au fil de l'eau (on a aussi imaginé diverses choses pour faire participer le public), on a quand même décidé de faire un tour de chauffe... Vous trouverez aussi en attaché les boucles pour chacune des entrées.

Vous suivez???

Je suis conviée à une nouvelle session de travail collective dans deux semaines, je soumettrai et défendrai mordicus notre idée, bien sûr, mais aucune assurance qu'ils marchent dans le jeu avec nous...

Voilà. En espérant que tout ça vous amuse encore autant une fois redescendus... Je suis en tout cas bien heureuse des moments d'échange en perspective!

Bises

Sara

Les jeux de ficelle, envoyons un jeu de ficelle sur la Lune. C'est revenu comme ça, un élastique qui claque au visage de celui qui a tiré trop fort dessus. Retour chariot.

Encore un flash-back de mes premières années d'étudiante en anthropologie. Ce professeur, un homme à l'élégance désuète qui correspondait tout à fait au professeur d'université que j'avais attendu impatiemment tout au long de mes années de lycée, nous avait parlé des jeux de ficelle. II nous avait expliqué que dès la fin du XIX $x^{e}$ siècle les anthropologues s'étaient intéressés à cette pratique observée un peu partout sur Terre, qui consiste à créer des images et des figures, en passant et repassant entre ses doigts une boucle de fil. Ces motifs, qui se forment au fur et à mesure de la narration, sont l'occasion de parler de sexualité, ils servent aussi d'aide-mémoire pour rappeler des règles sociales, à conter des histoires. Comme toujours, les jeux de ficelle ont fait l'objet de collectes, des figures classées, répertoriées, comparées. Toute ressemblance avec un projet lunaire... Je me souviens que les jeux de ficelle avaient été l'un des supports utilisés par les tenants de la théorie de la diffusion: les inventions sont peu nombreuses donc lorsqu'on rencontre des choses semblables dans des espaces distants, il y a fort à parier qu'elles se sont diffusées depuis le lieu de leur création. Ainsi, en suivant les transformations d'une même chose, on peut remonter le temps, suivre les routes empruntées par les migrations lointaines. Et puis les anthropologues 
s'étaient rendu compte que des choses que l'on pouvait penser semblables ne l'étaient pas tant que cela - on obtient une même figure de cordelette de bien des manières différentes - et que parfois aussi deux choses assez identiques pouvaient naître dans des lieux différents - le savoir des diffusionnistes a été mis en boîte mais ne gagnera sans doute jamais son ticket pour la Lune! Je me souviens enfin que notre professeur nous avait parlé de l'Association internationale du jeu de ficelle, fondée en 1978 par Hiroshi Noguchi, un mathématicien japonais, et Philip Noble, un missionnaire qui avait découvert ces tissages entre les doigts alors qu'il évangélisait en PapouasieNouvelle-Guinée, afin de rassembler, conserver et diffuser le savoir sur les jeux de ficelle. Toute ressemblance avec un projet lunaire... L'association a son siège à Pasadena, Californie. C'est tout ce dont je me rappelle. Dès le mot «Pasadena» prononcé, mon esprit s'est mis à vagabonder en Californie jusqu'à la fin de la séance.

Jusqu'à Eric Vandendriessche. Et puis Donna Haraway, Isabelle Stengers, qui ont fait des string figures une méthode de connaissance, un outil du savoir en train de se faire. Les ficelles et leurs figures sont des objets à penser, des objets pour penser. Les ficelles sont accueillantes. À tout moment, de nouveaux participants - quels qu'ils soient, quelle que soit leur espèce - peuvent entrer dans le jeu. II suffit qu'ils prêtent leurs doigts ou quoi que ce soit qui y ressemble pour participer.

La figure tissée est évolutive, le motif est imprévisible, mouvant, toujours susceptible de bifurquer. Les possibles sont infinis, le savoir se fait tentaculaire. Tout le monde peut s'approprier un jeu de ficelle, il s'échange, chacun y donne un sens. II suffit de ne jamais rien clore, de laisser le jeu ouvert, de garder une place à celui - à ce qui - voudra entrer; il suffit de changer le motif, d'avancer ensemble. Dans le jeu de ficelle, le sens n'est pas donné mais à créer; en cela, le jeu est ouvert à tous, à tout. Même la bactérie - car soyons sérieux: nos destinataires à dix mille ans peuvent-ils être autre chose que des bactéries? Et qu'est-ce que je leur dis, moi, à des bactéries? peut participer, en mangeant une couleur, en écornant une image. Au pire, on aura envoyé une méga-piste de danse pour bactéries: je les vois d'ici, guincher sur le dancefloor jusqu'au bout de la nuit lunaire.

C'est bien ça qu'il faudrait faire, c'est ce genre de tissage collectif qui mériterait de partir sur la Lune, un assemblage de fils qui interroge, qui donne envie d'y mettre les doigts, même à dix mille ans de là! II faudrait composer un panier, un pot, un filet à la Ursula Le Guin. Un contenant qui peut s'échanger, créer un motif qui passerait de main en main, qui se ferait en chemin. Composer une nébuleuse, une configuration qui demanderait à chacun de faire avec, de faire soi-même. Abandonnée, l'idée de la collection ordonnée; abandonnée, l'idée des fragments disparates et, dans le même temps, abandonné le point de vue sur, au sujet de... ces regards d'en haut, ces vues au microscope. Envoyons un essai, un modèle habité par ses participants. Ils auront essayé, raté, seront passés au suivant et auront laissé le jeu en plan, le jeu ouvert pour le suivant. Avec le jeu de ficelle, c'est moins l'accumulation ou l'agencement qui importe que les relations non explicitées entre des images que l'on se transmet de proche en proche selon des logiques à débusquer. Envoyons sur la Lune une devinette sans réponse, une énigme sans solution. 
Ce sont les correspondances, et toutes leurs potentialités d'embrayeurs de savoirs, les interstices, qui comptent. Envoyer quelque chose qui ne serait la synthèse de rien. Un cheminement, plutôt qu'un amoncellement.

\section{Les pieds sur Terre}

Le 15 décembre 2018 à 04:16:21 UTC+1, Loar Sara <saraloar@laposte.net> a écrit:

Chers toutes et tous,

Je sais, par les uns et par les autres, que les images ont commencé à circuler et que vous prenez l'exercice avec une certaine dubitation! Restent beaucoup de choses à caler et la session de travail avec Nicolas et les autres que nous avons eue il y a dix jours a continué d'en préciser certaines autres...

J'ai eu l'occasion déjà de raconter à plusieurs d'entre vous le gros de ces deux jours de session: le projet continue pour moi de se développer avec toute son inévidence, je dois dire, et pendant cette session j'ai dû batailler assez fort pour nous faire entendre. Je me dis que c'est bien, ça me forge, mais en même temps j'ai été prise plusieurs fois d'une violente envie de les envoyer faire un tour tout seuls sur la Lune...

L'équipe est très inquiète: notre dispositif va-t-il permettre de couvrir tout ce qu'ils avaient en tête pour l'anthropological team? II m'a fallu faire valoir que notre discipline (au sens large) comprenait sa propre complexité qui n'était pas soluble dans des questions simples comme la diversité de l'humanité et que, de manière générale, les «histoires » seraient plus intéressantes si elles posaient des questions (un peu comme des énigmes) plutôt que de viser le panorama exhaustif d'une situation. Ils sont d'accord (mais pas enthousiastes non plus) sur le principe mais, au fil des discussions, on est toujours à deux doigts d'embrayer sur l'encyclopédie, la collection, etc.

Bien... Je suppose qu'on peut encore un peu imaginer comment gripper les rouages du projet, complexifier autant que possible autour de questions qui nous intéressent... Mais je me rends bien compte que l'enthousiasme collectif du départ s'érode à mesure des frottements avec l'équipe...

Bises

Sara

Je n'arrive pas à dormir.

Cinq heures du matin.

Debout dans moins d'une heure quarante.

Par quelque bout que je la prenne, cette histoire est décidément inextricable, je peux hacker tout ce que je veux, je peux déjouer les pièges, voire les piéger, je peux me la jouer panier à la Ursula Le Guin, chasseusecueilleuse-rêveuse versus super-héros: rien n'y fait, c'est moi qui suis piégée. 
Assez colonisé comme ça: je jette l'éponge! J'ai déjà assez à faire avec les 104 ans de ma capsule temporelle familiale à vider sans fâcher personne avec quiconque - 104 ans de bazar accumulé dans cette baraque où le temps s'est à la fois figé et empilé, maison-à-vendre-avec-vuesur-la-mer-et-sur-les-polders comme le chantait Dick Annegarn. Je prends l'échelle pour descendre.

J'ai adoré le voyage. II me reste la boîte à savon bleu ciel et dorée. «Love You to the Moon and Back». C'est l'essentiel.

\section{Remerciements :}

Sara Loar remercie Sarah Carton de Grammont, Grégory Delaplace, Pierre-Olivier Dittmar, Sophie Houdart, Christine Jungen, Stefan Le Courant et Vanessa Manceron pour avoir contribué à la faire exister. Elle exprime également sa gratitude à Nicolas et son équipe; aussi à Vincent Debaene, Elsa De Smet, Mélanie Pavy et Istvan Praet; enfin, au domaine du Tournefou - ça ne s'invente pas qui a accueilli le collectif en résidence d'écriture en février 2018. 
Bibliographie

\author{
Barthes, Roland \\ 1980 La Chambre claire. \\ Note sur la photographie. Paris, \\ Éditions de l'Étoile/Gallimard/Seuil.
}

\section{Batut, Arthur}

1887 La photographie appliquée à la production du type d'une famille, d'une tribu ou d'une race. Paris,

Gauthier-Villars.

\section{Boym, Svetlana}

2001 The Future of Nostalgia. New York, Basic Books.

\section{Brunhes, Jean}

1913 «Ethnographie et géographie humaine", L'Ethnographie 1: 29-40.

\section{Jean-Brunhes}

Delamarre, Mariel et Beausoleil, Jeanne

1993 " Deux témoins de leur temps: Albert Kahn et Jean Brunhes", in Jean Brunhes. Autour du monde. Regards d'un géographe, regards de la géographie, catalogue d'exposition. Boulogne-Billancourt/ Paris, Musée Albert Kahn/Vilo: 91-107.

\section{Castro, Teresa}

2008 "Les Archives de la Planète et les rythmes de l'Histoire ", 1895. Mille huit cent quatre-vingt-quinze. Revue de l'association française de recherche sur l'histoire du cinéma $54:$ 56-81.

2011 "Les "Archives de la Planète" d'Albert Kahn ", in Christian Jacob (dir.), Les Lieux de savoir. Les Mains de l'intellect, t. II. Paris, Albin Michel: 877-895.

\section{D'Agata, John}

2012 [2010] Yucca Mountain trad. de l'anglais par S. Renaut. Bruxelles, Zones sensibles.

\section{D'Agata, John et Fingal, Jim}

2012 The Life Span of a Fact. New York/Londres, W.W. Norton.

\section{Danowski, Déborah} et Castro, Eduardo Viveiros de

2014 "L'arrêt de monde ", in Émilie Hache (dir.), De l'univers clos au monde infini. Bellevaux, Éditions Dehors.

Décapsuler. Une histoire vraie. Par Sara Loar

\section{Daston, Lorraine et Galison, Peter}

2012 [2007] Objectivité, trad. de l'anglais par S. Renaut et H. Quiniou. Paris, Les presses du réel.

\section{Descola, Philippe}

2001 Anthropologie de la nature. Paris, Collège de France, https:// books.openedition.org/cdf/1330

\section{Didi-Huberman, Georges}

2011 «Échantillonner le chaos. Aby Warburg et l'atlas photographique de la Grande Guerre ", Études photographiques 27.

\section{Falguières, Patricia}

2003 Les Chambres des merveilles. Paris, Bayard.

\section{Griaule, Marcel}

1957 Méthode de l'ethnographie. Paris, Presses universitaires de France.

\section{Haraway, Donna}

2016 Staying with the Trouble. Making Kin in the Chthulucene. Durham, Duke University Press.

\section{Hegland, Jean}

2017 [1995] Dans la forêt,

trad. de l'anglais par

J. Chicheportiche. Paris,

Gallmeister.

\section{Hergé}

1954 On a marché sur la Lune. Paris, Casterman.

\section{Hansen, Carla} et Hansen, Vilhelm

1973 Petzi chez les animaux de Lune. Paris, Casterman.

\section{Ingold, Tim}

2015 The Life of Lines. Londres/ New York, Routledge.

\section{Jansson, Tove}

1982 [1945] Une comète au pays de Moumine, trad. du suédois par K. et P. Chaplet. Paris, Librairie générale française.

\section{Jarvis, William E.}

2003 Time Capsules. A Cultural History. Jefferson, McFarland \& Co.
Joulian, Frédéric et Jeanjean, Agnès

2013 "Anthropologie évolutionnaire", Annuaire de l'EHESS: 400-402.

\section{Latour, Bruno}

2015 Face à Gaïa. Huit conférences sur le nouveau régime climatique.

Paris, La Découverte.

\section{Le Guin, Ursula K.}

1989 Dancing at the Edge of the World. New York, Grove Press.

\section{L'Estoile, Benoît (de)}

2008 "L'anthropologie après les musées? ", Ethnologie française 4 (38) : 665-670.

\section{Meillassoux, Quentin}

2013 Métaphysique et fiction des mondes hors-science. Paris, Aux forges de Vulcain.

\section{Perec, Georges}

1978 La Vie mode d'emploi. Paris, Hachette.

\section{Peyo et Delporte, Ivan}

1970 Le Cosmoschtroumpf. Paris, Dupuis.

\section{Polo, Marco}

2017 [1298] Le livre des merveilles du monde, trad. de I'italien par J.F. Kosta-Thefaine. Paris, Librio.

\section{Praet, Istvan}

2015 "La lune de Saturne et le "nous" œcuménique. Astrobiologie et anthropologie", trad. de l'anglais par C. Joseph, Gradhiva 22 : 136-167.

\section{Robic, Marie-Claire}

1993 "Jean Brunhes, un expert aux Archives de la Planète ", in Albert Kahn musée et jardins (dir.) Jean Brunhes. Autour du monde. Regards d'un géographe, regards de la géographie, catalogue d'exposition. Boulogne-Billancourt/ Paris, Musée AlbertKahn/Vilo: 109-137.
Sagan, Carl, Drake, Frank, Druyan, Ann, Ferris, Timothy, Lomberg, Jon, Salzman et Sagan, Linda

1978 Murmurs of Earth. The Voyager Interstellar Record. New York, Random House.

\section{Schnapper, Antoine}

1988 Collections et collectionneurs dans la France $d u \times v I^{\ominus}$ siècle.

Le Géant, la licorne et la tulipe, vol. I. Paris, Flammarion.

\section{Stengers, Isabelle}

2017 Civiliser la modernité? Whitehead et les ruminations du sens commun. Dijon,

Les presses du réel.

\section{Ungerer, Tomi}

2012 [1969] Jean de la Lune, trad. de l'anglais par A. Chagot. Paris, L'école des loisirs.

\section{Vandendriessche, Eric}

2015 String Figures as

Mathematics? An Anthropological Approach to String Figure-Making in Oral Tradition Societies. Heidelberg/ New York, Springer.

\section{Warburg, Aby}

2012 L'Atlas Mnémosyne, trad. de l'allemand par S. Zilberfarb. Dijon L'écarquillé.

\section{Yurchak, Alexei}

2008 « Post-Post-Communist Sincerity: Pioneers, Cosmonauts and Other Soviet Heroes Born Today ", in Thomas Lahusen et Peter Solomon (dir.), What is Soviet now? Identities, Legacies, Memories. Berlin, Lit.

1999 « Gagarin and the Rave-Kids: Transforming Power, Identity and Aesthetics in the Post-Soviet Nightlife ", in Adele Barker (dir.), Consuming Russia. Popular Culture, Sex, and Society since Gorbachev. Durham, Duke University Press. 
